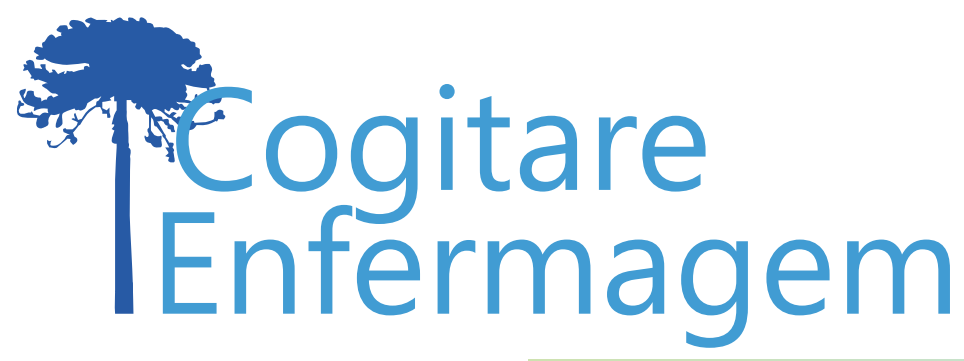

ARTIGO ORIGINAL

\title{
CONSTRUÇÃO COLETIVA DE UM FLUXOGRAMA PARA ACOMPANHAMENTO DAS GESTANTES COM SÍFILIS NO MUNICÍPIO DE SÃO JOSÉ-SC*
}

\begin{abstract}
Vanessa Beatriz da Silveira Silva1', Marli Terezinha Stein Backes², Janeide Freitas de Mello ${ }^{3}$, Jardel Scremin Magagnin ${ }^{4}$, Joyce Menezes Brasil ${ }^{5}$, Catarina Izabel da Silva ${ }^{6}$, Cleverson dos Santos ${ }^{7}$
\end{abstract}

\section{RESUMO}

Objetivo: instrumentalizar, com fluxograma e Procedimento Operacional Padrão, os enfermeiros da Atenção Primária à Saúde, a fim de monitorar os casos de sífilis gestacional.

Método: pesquisa de abordagem qualitativa, tipo pesquisa-ação, modalidade prático-educativa. Participantes: representantes da Vigilância Epidemiológica, Programa Saúde da Mulher, Atenção Primária à Saúde, enfermeiros da Estratégia Saúde da Família e do corpo clínico médico. Concretizado na prefeitura de São José-SC, no mês de outubro de 2017.

Resultados: elaborou-se um instrumento para auxiliar no atendimento de gestantes com exame de teste rápido reagente para sífilis.

Conclusão: os participantes atuantes na prática assistencial da atenção primária permitiram, através de um processo dinâmico, a legitimidade, buscando atender às demandas dos serviços e dos profissionais. A celeridade no diagnóstico, acompanhamento e tratamento da sífilis na gestação estão relacionados com o manejo adequado, associado a ações, estratégias e atualizações, proporcionando assistência qualificada durante o período gestacional, para efetivamente erradicar a sífilis congênita.

DESCRITORES: Sífilis Congênita; Atenção Primária à Saúde; Gestantes; Gestão em Saúde; Enfermagem.

*Artigo extraído da dissertação de mestrado "Monitoramento das gestantes com diagnóstico de sífilis pelo enfermeiro na atenção primária à saúde do município de São José/SC". Universidade Federal de Santa Catarina, 2019.

\section{COMO REFERENCIAR ESTE ARTIGO:}

Silva VB da S, Backes MTS, Mello JF de, Magagnin JS, Brasil JM, Silva Cl da, et al. Construção coletiva de um fluxograma para acompanhamento das gestantes com sífilis no município de São José-SC. Cogitare enferm. [Internet]. 2020 [acesso em "colocar data de acesso, dia, mês abreviado e ano"]; 25. Disponível em: http:// dx.doi.org/10.5380/ce.v25i0.65361.

\section{(c) (1)}

Este obra está licenciado com uma Licença Creative Commons Atribuição 4.0 Internacional.

${ }^{1}$ Enfermeira. Mestre em Gestão do Cuidado em Enfermagem. Enfermeira do Programa IST/HIV/Aids, Prefeitura de São José São José. São José, SC, Brasil. $\odot$

Enfermeira. Doutora em Enfermagem. Docente da Universidade Federal de Santa Catarina. Florianópolis, SC, Brasil. (ㅇ

${ }^{3}$ Enfermeira. Doutora em Enfermagem. Enfermeira do Hospital Universitário da Universidade Federal de Santa Catarina. Florianópolis, SC, Brasil. $($ )

${ }^{4}$ Enfermeiro. Especialista em Gestão em Enfermagem. Enfermeiro da família e coordenador de Unidade Básica de Saúde. Prefeitura de São José. São José, SC, Brasil. $\odot$

${ }^{5}$ Enfermeira. Especialista em Saúde da Mulher e aspectos Interdisciplinares. Enfermeira do Programa Saúde da Mulher. Prefeitura de São José. São José, SC, Brasil. $($ )

${ }^{6}$ Enfermeira. Especialista em Vigilância em Saúde. Enfermeira da Vigilância Epidemiológica do Programa IST/HIV/Aids, Prefeitura de São José. São José, SC, Brasil. +8

${ }^{7}$ Enfermeiro. Mestre em Gestão do Cuidado em Enfermagem. Enfermeiro do Programa IST/HIV/Aids. Prefeitura de São José. São José, SC, Brasil. 


\title{
COLLECTIVE CONSTRUCTION OF A FLOWCHART FOR FOLLOW-UP OF PREGNANT WOMEN WITH SYPHILIS IN THE MUNICIPALITY OF SÃO JOSÉ-SC
}

\begin{abstract}
Objective: To instruct, with a flowchart and Standard Operating Procedure, nurses from Primary Health Care, in order to monitor cases of gestational syphilis.

Method: Qualitative research, action-research type, practical-educational modality. Attendees: Representatives of the Epidemiological Surveillance, Women's Health Program,

Primary Health Care, nurses from the Family Health Strategy and the medical staff. Made in the city of São José-SC, in October 2017.

Results: An instrument was developed to assist pregnant women with a rapid reagent test for syphilis.

Conclusion: The participants working in the primary care assistance practice, through a dynamic process, allowed legitimacy, seeking to meet the demands of the services and the professionals. The speed in the diagnosis, monitoring and treatment of syphilis in pregnancy are related to the proper management, associated with actions, strategies and updates, providing qualified assistance during the gestational period, to effectively eradicate congenital syphilis.
\end{abstract}

DESCRIPTORS: Congenital Syphilis; Primary Health Care; Pregnant Women; Healthcare Management Nursing.

\section{CONSTRUCCIÓN COLECTIVA DE UN FLUJOGRAMA PARA SEGUIMIENTO DE GESTANTES CON SIFILIS EN EL MUNICIPIO DE SAN JOSÉ-SC}

\begin{abstract}
RESUMEN
Objetivo: instrumentalizar, con flujograma y Procedimiento Operativo Estándar, a los enfermeros de la Atención Primaria de la Salud, con el objetivo de monitorear los casos de sífilis gestacional.

Método: investigación de enfoque cualitativo, tipo investigación-acción, modalidad prácticoeducativa. Participantes: representantes de la Vigilancia Epidemiológica, Programa Salud de la Mujer, Atención Primaria de la Salud, enfermeros de la Estrategia Salud de la Familia y del cuerpo de clínica médica. Estudio realizado en la municipalidad de San José-SC, durante el mes de octubre de 2017.

Resultados: se elaboró un instrumento para auxiliar en la atención de gestantes con de test rápido reactivo para sífilis.

Conclusión: los participantes actuantes en la práctica asistencial da atención primaria permitieron, por medio de un proceso dinámico, la legitimidad, intentando atender las demandas de los servicios y de los profesionales. La celeridad en el diagnóstico, seguimiento y tratamiento de la sífilis gestacional se relacionan con el manejo adecuado, asociado a acciones, estrategias y actualizaciones, brindando asistencia cualificada durante el período gestacional, para erradicar la sífilis congénita de manera efectiva.
\end{abstract}

DESCRIPTORES: Sífilis Congénita; Atención Primaria de la Salud; Gestantes; Gestión en Salud; Enfermería. 
A sífilis é uma Infecção Sexualmente Transmissível, considerada um grande problema de saúde pública pela Organização Mundial da Saúde, apesar de apresentar diagnóstico e tratamento bem estabelecidos e de baixo custo. Uma das principais preocupações sobre as dificuldades no controle da disseminação dessa doença é a infecção de mulheres em idade reprodutiva, que pode acarretar na ocorrência de casos de sífilis congênita, por meio da transmissão vertical ${ }^{(1)}$.

A cada ano, quase seis milhões de pessoas são infectadas pela sífilis. Uma das causas para o acometimento da população é o não uso dos preservativos, associado ao comportamento de risco de rotatividade de parceiros ${ }^{(2)}$. No Brasil, no ano de 2015, 33.365 gestantes adquiriram sífilis, sendo que $6.005(18,0 \%)$ pertencem à região Sul do país ${ }^{(3)}$. Em Santa Catarina, o número de casos também é significativo. Em 2016, notificou-se 1.380 gestantes com sífilis, atingindo 554 bebês e causando a morte de 34 destes, além de 18 abortos $^{(4)}$.

De modo a propiciar o tratamento precoce da sífilis e possibilitar o início em tempo oportuno, novas estratégias são adotadas pelo Ministério da Saúde (MS) com o objetivo de ampliar o diagnóstico e introduzir novas metodologias e fluxos que permitam o tratamento precoce da sífilis. Dentre as inovações propostas, está o diagnóstico por meio de testes rápidos. Trata-se de testes de fácil execução e leitura simples, possibilitando a investigação da sífilis em locais sem infraestrutura laboratorial e, muitas vezes, de difícil acesso. Além disso, por gerar resultados em até 30 minutos, os testes rápidos eliminam o risco do usuário não voltar ao sistema de saúde para saber seu resultado e, desta forma, possibilitam o seguimento imediato do indivíduo ${ }^{(5)}$.

A sífilis pode ser classificada em primária, secundária, latente recente, e latente tardia ou terciária. Sífilis primária refere-se ao diagnóstico no período de 10 a 90 dias após o contato, a secundária ao período de seis semanas a seis meses após contato, latente recente até o período de dois anos e latente tardia o período após dois anos do contato(6).

Diante de uma gestante com diagnóstico de sífilis confirmado já no teste rápido, em situações em que não é possível entender a duração da infecção, classifica-se sempre como sífilis latente tardia, fazendo o tratamento com dose total de penicilina ${ }^{(6)}$. Desta forma, o município do estudo opta em seguir o mesmo protocolo do Ministério da Saúde, com inclusão do tratamento estendido ao parceiro da gestante, mesmo sem realizar o exame de sífilis.

O MS recomenda que os casos de sífilis adquirida em gestantes sejam acompanhados mensalmente ${ }^{(5)}$. Ademais, a notificação compulsória de sífilis congênita em todo o território nacional foi instituída por meio da Portaria $n^{\circ} 542$, de 22 de dezembro de 1986; a de sífilis em gestante foi instituída pela Portaria $n^{\circ} 33$, de julho de 2005; e, por último, a de sífilis adquirida, por intermédio da Portaria $n^{\circ} 2.472$, publicada em 31 de agosto de $2010^{(3)}$.

Devido a esta obrigatoriedade de notificação das gestantes infectadas ainda no prénatal, o MS busca cada vez mais estratégias que otimizem este cuidado. Em 2012, instituiu a realização dos testes rápidos para HIV, sífilis e hepatites $\mathrm{B}$ e $\mathrm{C}$ em todas as Unidades Básicas de Saúde (UBS) para as gestantes no primeiro trimestre, buscando em momento oportuno o início do tratamento, coibindo a transmissão vertical(6).

O município de São José-SC dispõe do Programa Saúde da Mulher, o qual possui um protocolo de assistência integral à mulher e à criança, com o intuito de atualizar os profissionais quanto ao atendimento e tratamento no âmbito da Atenção Primária à Saúde, juntamente com a vigilância epidemiológica do município. Desde 2014, o município busca otimizar 0 atendimento às gestantes com o teste rápido. Inicialmente, foram ofertados os testes no Centro de Testagem e Aconselhamento. Após um ano da implantação dos testes rápidos, eles foram disponibilizados nas UBS. Até o presente momento, todas as UBS dispõem de testes rápidos para diagnóstico do HIV, triagem da sífilis, hepatite B e C. 
O uso de ferramentas de gestão e de atenção para o cuidado da gestante com diagnóstico de sífilis, quando bem elaborados e implementados com as equipes de saúde devidamente capacitadas para tal, possibilitam a erradicação da sífilis congênita. Desta maneira, optou-se por construir duas ferramentas, uma de gestão - fluxograma - e outra de atenção - Procedimento Operacional Padrão (POP) - para auxiliar a equipe de saúde na tomada de decisão frente a este problema de saúde. Deste modo, o objetivo desta pesquisa consiste em instrumentalizar, com fluxograma e POP, os enfermeiros da Atenção Primária à Saúde, a fim de que monitorem os casos de sífilis durante a gestação.

\section{MÉTODO}

Pesquisa de abordagem qualitativa do tipo pesquisa-ação, na modalidade de prática educativa, onde se construiu um método de monitoramento por meio de um fluxograma e de um POP para os casos de diagnóstico de sífilis em gestantes. Estas ferramentas serão incorporadas ao Protocolo Assistencial da Saúde da Mulher do Município de São José-SC, e utilizadas pelos enfermeiros da Estratégia Saúde da Família (ESF).

A pesquisa-ação, partindo das situações vivenciadas no cotidiano, possibilita refletir a prática assistencial, conduzindo para descobrir novas realidades e introduzir inovações na prática assistencial. Consiste na articulação intencional com a prática e as ações de assistência, que vão sendo incorporadas no processo de pesquisa e vice-versa. A pesquisaação requer sempre a participação ativa dos sujeitos que farão parte da pesquisa e está orientada para a realização de mudanças e/ou introdução de inovações nas práticas de saúde. Portanto, é compreendida e realizada em articulação com as ações que envolvem pesquisadores e demais pessoas representativas da situação a ser pesquisada, em uma relação de cooperação mútua(7).

A ação educativa, no âmbito da educação em saúde, torna-se um processo dinâmico cujo objetivo é a capacitação dos indivíduos e/ou grupos em busca da melhoria das condições de saúde e de trabalho(8). Pensando neste processo de construção coletiva, incluiu-se nessa pesquisa a equipe do Programa Saúde da Mulher e da vigilância epidemiológica. $\mathrm{Na}$ ocasião da atividade, os representantes dos programas indicaram um enfermeiro da ESF de cada distrito sanitário, cabendo ao coordenador selecionar, por seu critério de experiência profissional, para colaborar nas ideias de construção da ferramenta de gestão, por estarem envolvidos diretamente no processo de atendimento das gestantes no pré-natal.

Aos participantes foi entregue um convite pessoalmente pela pesquisadora. Compareceram na atividade educativa os representantes da atenção básica, da vigilância epidemiológica, do Programa Saúde da Mulher, o médico e os enfermeiros da ESF dos distritos sanitários sul, leste e oeste, totalizando oito participantes. Não compareceu a enfermeira da ESF do distrito sanitário norte.

A atividade educativa aconteceu no dia 18 de outubro de 2017, com duração de uma hora e 40 minutos, no auditório da Secretaria Municipal de Educação. A sala foi organizada em círculo, possibilitando que os participantes pudessem se olhar durante a prática. A ação foi realizada pela pesquisadora, e iniciou com a apresentação da temática, levantando a percepção do problema e argumentando por meio das estatísticas crescentes o porquê da necessidade de estratégias para minimizar os danos da sífilis na gestação, na ausência de tratamento adequado. Para justificar que já existem portarias que visam este cuidado, foi apresentada a portaria ministerial e o protocolo do município, à vista da assistência deficitária nos casos de gestantes com teste rápido de sífilis reagente.

Para iniciar o processo de elaboração do fluxograma e do POP, apresentaram-se as informações que precedem a construção dos elementos necessários em ambos os documentos. No caso do POP, o município já dispunha de um modelo padrão, sendo necessário realizar um específico para a gestante com diagnóstico de sífilis. Já o que 
concerne ao fluxograma, foram apresentadas as etapas que definem os componentes do processo, a importância da ordem das figuras geométricas, que definem tanto a continuação como a finalização do acompanhamento, e a justificativa da elaboração dos mesmos.

As sugestões foram corroboradas por todos, e finalizou-se justamente com a mesma opinião, não tendo dúvidas ou divergências. Posteriormente, apresentou-se a proposta por meio de capacitações aos enfermeiros da UBS, sendo este revisado e aprovado.

O estudo foi aprovado pelo Comitê de Ética em Pesquisa com Seres Humanos da Universidade Federal de Santa Catarina, parecer $n^{\circ}$ 2.836.404.

\section{RESULTADOS}

Com a realização da atividade educativa, focando na capacitação dos indivíduos para que busquem formas de melhorar os processos de trabalho, buscou-se a coletividade de conhecimentos, estimulando o diálogo e a reflexão. A participação dos profissionais foi indispensável, visto que estes atuam no campo da prática, conhecem as reais necessidades e conseguem priorizar as carências dos usuários. Assim, a aprendizagem se deu de maneira efetiva, pois o grupo teve a oportunidade de compartilhar ideias, receber apoio, promover um sentimento de companheirismo, reforçando o conhecimento oriundo de seus colegas de trabalho. De forma coletiva, dois produtos resultaram da prática educativa, um fluxograma e um POP, apresentados a seguir.

Em outubro de 2017, foi realizada uma ação educativa no auditório norte da Secretaria Municipal de Educação da prefeitura de São José-SC. Participaram os representantes da Atenção Primária, Vigilância Epidemiológica, Programa Saúde da Mulher, um enfermeiro da ESSF de cada distrito sanitário do município e um médico, com o objetivo de uma construção coletiva de um POP com fluxograma, para posteriormente serem apresentados como ferramentas de gestão para a atenção ao cuidado das gestantes com diagnóstico de sífilis assistidas pelos enfermeiros da ESF.

Conhecido como uma representação gráfica de conteúdo, o fluxograma é um processo que utiliza de símbolos geométricos para descrever o passo a passo de um fluxo. O objetivo é mostrar de forma descomplicada as etapas das informações e elementos, além da sequência operacional que caracteriza o trabalho que está sendo executado.

O fluxograma desenvolvido nesse estudo visa orientar o profissional da assistência na condução da gestante ao realizar o teste rápido para sífilis, como observado na Figura 1. 


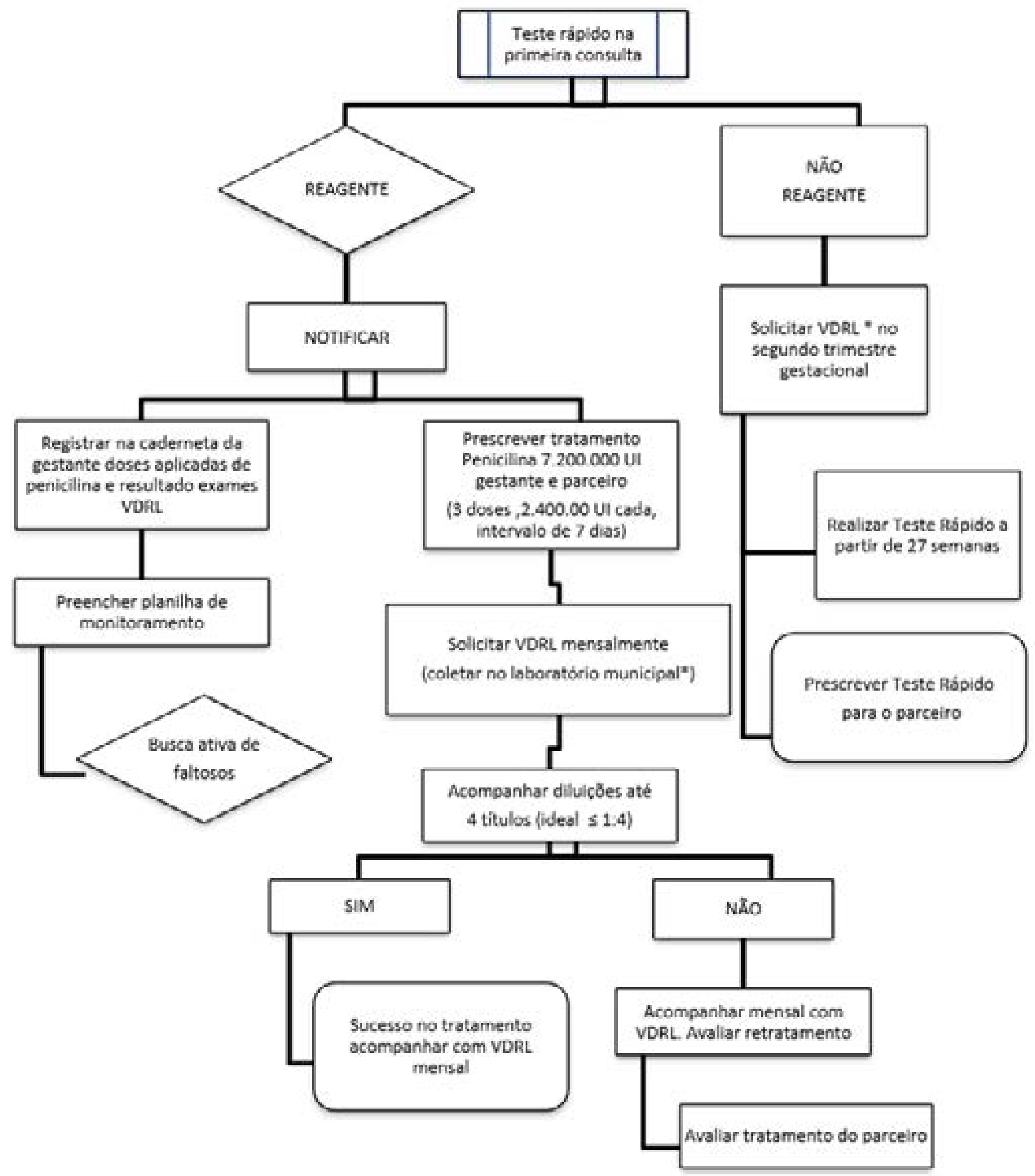

*Laboratório Municipal: Policlínica Forquilhinha. Horário de coleta das 7:00 as 17:00 de segunda a sexta-feira.

Legenda: $\square$ Processo pré-definido $\square$ Processo $>$ Exige tomada de decisão $\square$ Finalizador

Figura 1 - Fluxograma da gestante na realização do teste rápido para sífilis. São José, SC, Brasil, 2018

Por sua vez, o POP, pensado como uma meta padrão, é um documento que expressa o planejamento do trabalho repetitivo, que deve ser executado pela equipe de trabalho, de forma a padronizar os serviços. O POP construído pelos profissionais, nesta pesquisa, diz respeito ao acompanhamento das gestantes em tratamento de sífilis, explicitado no Quadro 1. 
Quadro 1 - Procedimento Operacional Padrão: Acompanhamento das gestantes em tratamento de sífilis. São José, SC, Brasil, 2018

\section{ACOMPANHAMENTO DAS GESTANTES EM TRATAMENTO DE SÍFILIS}

Data da criação: $18 / 10 / 2017$

Data da revisão: $31 / 10$ e 01/11 de 2018

Revisado por: Enfermeiros da ESF

Agente(s): Enfermeiro

Objetivo: realizar o monitoramento adequado das gestantes com diagnóstico de sífilis.

1. CONCEITO: As gestantes em tratamento de sífilis devem ser monitoradas mensalmente com testes não treponêmicos (VDRL - quantitativo). Considerando a detecção de possível indicação de retratamento, devido à possibilidade de falha terapêutica(1).

Gestantes em tratamento de baixo risco com teste rápido reagente para sífilis, realizar controle mensal para tratamento e cura(2).

\section{MATERIAIS NECESSÁRIOS}

Materiais de proteção individual

Kits de testes rápidos

\section{ETAPAS DO PROCEDIMENTO}

1. Agendar a consulta de pré-natal, preferencialmente até 12 semanas de gestação;

2. Primeira consulta de pré-natal com Enfermeiro da ESF;

3. Realizar teste rápido na primeira consulta de pré-natal, aconselhamento pré e pós teste;

4. Teste rápido reagente para sífilis: prescrever penicilina 7.200.000 UI para ser aplicada em três semanas, 2.400.000 UI a cada sete dias;

5. Aplicar primeira dose de penicilina 2.400.000 UI;

6. Solicitar VDRL quantitativo a cada 30 dias até o final da gestação;

7. Solicitar teste rápido para o parceiro;

8. Prescrever o tratamento do parceiro, 7.200.000 UI para ser aplicada em três semanas, 2.400.000 UI a cada sete dias (todos os parceiros devem ser tratados independentemente de realizarem a testagem); 9. Preencher planilha de monitoramento web (ferramenta institucional); 10. NOTIFICAR.

\section{REFERÊNCIAS}

1. Ministério da Saúde (BR). Secretaria de Vigilância em Saúde. Departamento de IST/HIV/ Aids e Hepatites Virais. Manual Técnico para Diagnóstico da Infecção pelo HIV. 3 ed. Brasília; 2016.

2. Prefeitura de São José. Santa Catarina (BR). Secretaria Municipal de Saúde. Diretoria de Vigilância Epidemiológica. Programa DST/HIV/AIDS/Hepatites Virais. Aconselhamento em DST/HIV/AIDS no Município de São José - SC. São José; 2015.

Colocar em prática um trabalho pautado na responsabilidade ética e que zele pela promoção da saúde, prevenção de doenças e agravos, configura uma das melhores estratégias para minimizar os casos de sífilis na gestação. Sendo a sífilis uma patologia de fácil e rápido diagnóstico e tratamento, a descoberta durante o pré-natal configura um desafio enorme para a atenção primária, porque geralmente as abordagens vem sendo individualizadas pelos profissionais, o que acarreta uma sucessão de erros no controle e tratamento da gestante e parceiro. A padronização do cuidado, como ferramentas de gestão, estabelece critérios coletivos, o que certamente oferece maior segurança ao profissional em estar realizando um serviço de qualidade sob a ótica assistencial.

A melhor forma de iniciar a padronização de um objeto é por meio da compreensão de como ocorre todo o processo e, neste caso, é necessária uma representação sistematizada. O POP é uma ferramenta que descreve cada passo crítico e sequencial, que deverá ser 
dado pelo operador, para garantir o resultado esperado da assistência. Além de relacionarse à técnica, refere-se à ajuda de uma regra para realizarmos algo com disposição(9).

A característica de desorganização dos serviços de saúde e assistência de enfermagem está pautada na falta de padronização dos procedimentos, inexistência de normas e rotinas e a não utilização de metodologia de serviços de enfermagem, devido às diferentes formas de conduta profissional. Por isso os padrões são definidos, visando ao estabelecimento de diretrizes para o controle e melhoria contínua da qualidade. Além disso, os cuidados padronizados são diretrizes detalhadas que representam o atendimento previsível, indicado para situações específicas, que irão impulsionar as organizações para o desenvolvimento da melhoria de seus processos e garantia de resultados ${ }^{(10)}$.

Enfermeiros bem capacitados propiciam racionalização de rotinas, padronização e maior segurança na realização dos procedimentos, participação efetiva no planejamento e liberação de mais tempo para interagir com o paciente e, desta forma, há a necessidade de acompanhar as novas determinações e participar da construção de alternativas que respondam aos desafios de melhorar a oferta de qualidade dos serviços prestados ${ }^{(11)}$.

O conteúdo do POP, assim como sua aplicação, deverá ter o completo entendimento e familiarização por parte da equipe de enfermagem que tenha participação direta e/ou indireta na qualidade final daquele procedimento, sendo o teste rápido realizado pelo enfermeiro na primeira consulta de pré-natal. Normalmente, a ingerência de supervisores, coordenadores e diretores neste ponto é uma das causas de ineficiência na implantação de um sistema da qualidade. Cabe aos mesmos as responsabilidades pela revisão e aprovação do $\mathrm{POP}^{(12)}$.

O POP se apresenta como base para garantir a padronização de tarefas e assegurar aos usuários um serviço livre de variações indesejáveis na sua qualidade final. Facilita o trabalho de todos que utilizarão este procedimento no dia a dia, proporcionando mais segurança aos enfermeiros e aos que utilizarão este serviço, sendo que todos ganharão com mais qualidade na assistência.

A palavra "padrão" tem como significado "aquilo que serve de base ou norma para a avaliação", e está relacionada aos resultados que se deseja alcançar. Na área da saúde, equivale aos padrões de cuidado e assistência, que se relacionam com os direitos do cliente de receber a assistência adequada, de acordo com as suas necessidades ${ }^{(12)}$.

O fluxograma, junto ao POP, convém para o enriquecimento da ferramenta de gestão e facilitador da gerência de atendimento, por descrever, através do uso de símbolos específicos, cada etapa de um processo. Esta ferramenta tem como objetivo descrever de forma clara um fluxo, sequência ou processo de trabalho, auxiliando, com isso, a criação de novos processos, o melhoramento de processos já existentes, a eliminação de tarefas em duplicidade, a eliminação de estragos/desperdícios de tempo e insumos, além de transmitir uma visão sistêmica de processos existentes na organização a todos os indivíduos envolvidos direta ou indiretamente na atividade representada ${ }^{(13)}$.

De forma analítica, o fluxograma é uma ferramenta que representa a rotina de um processo, sendo representadas as operações de início, deslocamentos, armazenamentos, esperas, inspeções, interrupções e conclusões deste processo, assim como representa o fluxo de informações e as consequências decorrentes da escolha da atuação ou não em determinado momento do processo pelo executor. Para construção de um fluxograma, necessariamente deve-se definir sua aplicação, identificar o início e o fim pretendido e - passo a passo das etapas, analisar se, de fato, replicam a realidade do processo ou atividade, usar os símbolos de maneira padronizada para facilitar o entendimento de todos os que terão acesso ao documento, e, sempre que necessário, analisar e atualizar, a fim de adaptar possíveis desvios ou alterações. O fluxograma é um grande facilitador, permitindo uma melhor visualização e maior entendimento das entradas, saídas, relações, interações e decisões ${ }^{(14)}$.

O fluxograma propicia o entendimento de qualquer alteração que se proponha 
nos sistemas existentes pela clara visualização das modificações introduzidas. Dentre suas vantagens, destaca-se que permite verificar como se conectam e relacionam os componentes de um sistema, mecanizado ou não, facilitando a análise de sua eficácia e a localização das deficiências, pela fácil visualização dos passos, transportes, operações e formulários. O fluxograma é apontado, graficamente, como o coração do mapeamento de processos, sendo frequentemente utilizado para fins de processamento de informações ${ }^{(14)}$.

Um instrumento que norteie o monitoramento dos casos de sífilis nas gestantes implica em elevar a qualidade da assistência de enfermagem, beneficiando tanto o paciente como o enfermeiro, validando o conhecimento científico da enfermagem, determinando a autonomia do enfermeiro, proporcionando uma melhor qualidade da assistência, tornando-a mais segura, qualificada e conferindo o aumento da satisfação e crescimento do profissional enfermeiro e, acima de tudo, a redução de sífilis congênita.

A justificativa da instrumentalização para o cuidado é ampliar a compreensão do quão importante é o papel do enfermeiro em prestar o cuidado adequado, observando a necessidade cada vez mais presente do uso de instrumentos que buscam melhorar a qualidade do cuidado prestado pelos enfermeiros e sua equipe aos usuários. $O$ trabalho em equipe é importante para a realização dessas mudanças, além de ser um caminho para a tomada de decisões conjuntas, pois sistematiza o cuidado e aproxima o enfermeiro do usuário, fortalecendo a presença indispensável deste profissional e contribuindo para o processo de reabilitação e cura dos indivíduos.

A prática educativa é uma ação do profissional enfermeiro e faz parte do cuidado em enfermagem. Desta forma, o conhecimento é considerado emancipador, pensa a consequência de seus atos, no qual a relação sujeito-objeto é substituída pela reciprocidade entre os participantes e onde a solidariedade e a participação estão presentes. Realizar educação em saúde em um processo que estimule a indagação, o diálogo, a reflexão e a ação partilhada, eleva o saber dos participantes. Para que a educação em saúde aconteça, é indispensável que os educadores conheçam a realidade, a visão de mundo e as expectativas de cada indivíduo, para que possam priorizar as necessidades dos usuários, e não apenas as exigências terapêuticas ${ }^{(8)}$.

Ressaltamos que o estudo se limitou a um contexto específico, sendo as ferramentas estruturadas às necessidades de saúde referentes a determinada população e à rede de saúde do município em questão. No entanto, estimamos que essa pesquisa possa servir de base para o desenvolvimento de novas pesquisas que busquem a instrumentalização dos profissionais, principalmente no que concerne à prevenção e tratamento de sífilis adquirida em gestantes e congênita.

\section{CONSIDERAÇÕES FINAIS}

Esta pesquisa visa contribuir na agilidade do diagnóstico, acompanhamento e tratamento da sífilis na gestante e parceiro, entendida como um ponto de suma importância, uma vez que o desconhecimento do manejo adequado a estas gestantes e parceiros está diretamente associado a ações e estratégias que necessitam ser repensadas por meio de atualizações e novidades que proporcionem assistência qualificada durante o período gestacional, para efetivamente erradicar a sífilis congênita.

Assim, o fluxograma e o POP, desde que bem elaborados e entendidos como ferramenta de gestão, tornam-se um apoio ao processo de tomada de decisões, pois seguem um fluxo único, corroborando com a busca do atendimento adequado, tanto por parte do profissional de saúde como para o tratamento e cura da sífilis ainda na gestação, evitando o nascimento de crianças com sífilis congênita. 
1. Ministério da Saúde (BR). Secretaria de Políticas de Saúde. Coordenação Nacional de Doenças Sexualmente Transmissíveis e Aids. Manual e controle das doenças sexualmente transmissíveis. Brasília: Ministério da Saúde; 2006 [acesso em 24 out 2018]. Disponível em: http://bvsms.saude.gov.br/bvs/ publicacoes/manual controle das dst.pdf.

2. Ministério da Saúde (BR). Secretaria de Vigilância em Saúde. Departamento de DST, Aids e hepatites virais. Diagnóstico laboratorial de doenças sexualmente transmissíveis, incluindo o vírus da imunodeficiência humana. Brasília: Ministério da Saúde; 2017 [acesso em 17 out 2018]. Disponível em: https://apps.who.int/iris/bitstream/handle/10665/85343/9789241505840 por. pdf;jsessionid=C03BCBCF70E5E98EAEFF6DD09E7A9A9A? sequence $=7$.

3. Ministério da Saúde (BR). Secretaria de Vigilância em Saúde. Boletim Epidemiológico. 47(35). Brasília: Ministério da Saúde; 2016 [acesso 21 out 2018]. Disponível em: http://portalarquivos2.saude.gov.br/ images/pdf/2016/outubro/31/2016 030 Sifilis-publicao2.pdf.

4. Santa Catarina (BR). Secretaria de Estado da Saúde. Diretoria de Vigilância Epidemiológica (DIVE). Notificações de casos de sífilis em Santa Catarina aumentam quase 50\% em 2015. Santa Catarina; 2016 [acesso em 20 out 2018]. Disponível em: http://www.dive.sc.gov.br/index.php/arquivo-noticias/271notificacoes-de-casos-de-sifilis-em-santa-catarina-aumentam-quase-50-em-2015.

5. Ministério da Saúde (BR). Secretaria de Vigilância em Saúde. Departamento de Vigilância, Prevenção e Controle das Doenças Sexualmente Transmissíveis, AIDS e Hepatites Virais. Manual técnico para diagnóstico da sífilis. Brasília: Ministério da Saúde; 2016 [acesso em 18 out 2018]. Disponível em: http:// www.aids.gov.br/pt-br/pub/2016/manual-tecnico-para-diagnostico-da-sifilis.

6. Ministério da Saúde (BR). Secretaria de Vigilância em Saúde. Departamento de Vigilância, Prevenção e Controle das Infecções Sexualmente Transmissíveis, do HIV/Aids e Hepatites Virais. Protocolo clínico e diretrizes terapêuticas para prevenção da transmissão vertical, sífilis e hepatites virais. Brasília: Ministério da Saúde; 2018 [acesso em 15 out 2018]. Disponível em: http://www.aids.gov.br/pt-br/pub/2015/ protocolo-clinico-e-diretrizes-terapeuticas-para-prevencao-da-transmissao-vertical-de-hiv.

7. Thiollent M. Metodologia da pesquisa-ação. 17. ed. São Paulo: Cortez, 2009.

8. Acioli S. A prática educativa como expressão do cuidado em Saúde Pública. Rev. bras. enferm. [Internet]. 2008 [acesso em 13 set 2017]; 61(1). Disponível em: http://dx.doi.org/10.1590/S003471672008000100019 .

9. Antunes AV, Trevizan MA. Gerenciamento da qualidade: utilização no serviço de enfermagem. Rev. Latino-Am. Enfermagem [Internet]. 2000 [acesso em 13 set 2017]; 8(1). Disponível em: http://dx.doi. org/10.1590/S0104-11692000000100006.

10. Silva VEF. Manuais de enfermagem. In: Kurcgant P. coordenadora. Administração em enfermagem. São Paulo: Editora Pedagógica e Universitária; 2016.

11. Magalhães AMM, Duarte ERM. Tendências gerenciais que podem levar a enfermagem a percorrer novos caminhos. REBEN [Internet]. 2004 [acesso em 27 jul 2018]; 57(4). Disponível em: http://dx.doi. org/10.1590/S0034-71672004000400004.

12. Guerrero GP, Beccaria LM, Trevizan MA. Procedimento operacional padrão: utilização na assistência de enfermagem em serviços hospitalares. Rev. Latino-Am. Enfermagem [Internet]. 2008 [acesso em 27 jul 2017]; 16(6). Disponível em: http://dx.doi.org/10.1590/S0104-11692008000600005.

13. Silveira CB. Fluxograma de processo: o que é, como elaborar e benefícios [Internet]. São Paulo; 2006 [acesso em 13 set 2017]. Disponível em: https://citisystems.com.br/fluxograma/.

14. Mello AENS de. Aplicação do mapeamento de processos e da simulação no desenvolvimento de projetos de processos produtivos [dissertação]. Itajubá (MG): Universidade Federal de Itajubá; 2008.

Disponível em: http://repositorio.unifei.edu.br/xmlui/handle/123456789/1695. 
Recebido: $12 / 03 / 2019$

Finalizado: 11/02/2020

Autor Correspondente:

Vanessa Beatriz da Silveira Silva

Universidade Federal de Santa Catarina

R. Professora Maria Flora Pausewang, s/nº 88040-900 - Florianópolis, SC, Brasil

E-mail: vanessabiasilveira@yahoo.com.br

Contribuição dos autores:

Contribuições substanciais para a concepção ou desenho do estudo; ou a aquisição, análise ou interpretação de dados do estudo - JFM, JMB

Elaboração e revisão crítica do conteúdo intelectual do estudo - MTSB

Aprovação da versão final do estudo a ser publicado - MTSB

Responsável por todos os aspectos do estudo, assegurando as questões de precisão ou integridade de qualquer parte do estudo - VBSS, JSM, CIS, CS 